\title{
Has the COVID-19 outbreak changed the way we are treating prostate cancer? An EAU - YAU Prostate Cancer Working Group multi-institutional study
}

\author{
Fabio Zattoni, ${ }^{1,2}$, Giancarlo Marra ${ }^{3}$, Alexander Kretschmer ${ }^{4}$, Felix Preisser ${ }^{5}$, Derya Tilki ${ }^{6}$, \\ Claudia Kesch7, Jan Philipp Radtke7, Nils Hoffmann7, Alessandro Morlacco², Fabrizio Dal Moro², \\ Timo F.W. Soeterik ${ }^{8}$, Roderick C.N. van den Bergh ${ }^{8}$, Francesco Barletta ${ }^{9}$, Alberto Briganti ${ }^{9}$, \\ Francesco Montorsi ${ }^{9}$, Giorgio Gandaglia9 ${ }^{9}$ on behalf of the EAU-YAU Prostate Cancer Working Group \\ ${ }^{1}$ Urology Clinic, Academical Medical Centre Hospital, Udine, Italy \\ ${ }^{2}$ Department of Surgical, Oncological and Gastroenterological Sciences-Urological Clinic, University of Padua, Padua, Italy \\ ${ }^{3}$ Department of Urology, Institut Mutualiste Montsouris, Paris, France \\ ${ }^{4}$ Department of Urology, Ludwig-Maximilians University of Munich, Munich, Germany \\ ${ }^{5}$ Department of Urology, University Hospital Frankfurt, Frankfurt, Germany \\ ${ }^{6}$ Martini-Klinik Prostate Cancer Center, and Department of Urology, University Hospital Hamburg-Eppendorf, Hamburg, Germany \\ ${ }^{7}$ Department of Urology, University Hospital Essen, Essen, Germany \\ ${ }^{8}$ Department of Urology, St Antonius Hospital, Utrecht, The Netherlands \\ ${ }^{9}$ Division of Oncology/Unit of Urology, Urological Research Institute, IRCCS Ospedale San Raffaele, Milan, Italy
}

Citation: Zattoni F, Marra G, Kretschmer A, et al. Has the COVID-19 outbreak changed the way we are treating prostate cancer? An EAU - YAU Prostate Cancer Working Group multi-institutional study. Cent European J Urol. 2021; 74: 362-365.

Article history

Submitted: Aug. 25, 2021

Accepted: Sept. 5, 2021

Published online: Sept. 18, 2021

Corresponding author

Fabio Zattoni

ORCID ID: 0000-0002-

4178-373X

University of Padova

Oncological and

Gastroenterological

Sciences-Urological Clinic

Department of Surgery

35128 Padova, Italy

phone: +39 0498212720

fabiozattoni@gmail.com
Introduction The COVID-19 outbreak has become the dominant issue throughout the world whilst the governments, nations and health services are trying to deal with its impact. The aim of our study is to assess the impact of COVID-19 on patients treated with radical prostatectomy (RP) for prostate cancer (PCa) at European referral centers in terms of surgical volume (SV), waiting list meant as time from biopsy to surgery (WL) and risk of adverse pathologic findings at RP due to the selection of men with more adverse disease characteristics at final pathology.

Material and methods Consecutive patients with a diagnosis of histologically proven PCa treated with RP between March 2020 (WHO declaration of pandemic) and December 2020 were identified. Patients with metastatic disease not eligible to local treatment and recurrent prostate cancer after RP or RT were excluded. Patients treated at the same institutions between March 2019 and December 2019 were considered as the control group. Multivariable logistic regression analysis tested the impact of the COVID-19 outbreak on the risk of adverse pathologic findings at RP after adjusting for confounders. The percentage change of SV and WL was assessed comparing the months of pandemic with the equivalent timespan of the previous year.

Results A total of 2,574 patients treated with RP (927 cases and 1647 controls) were identified in 8 European tertiary referral centers. At multivariable analysis patients who were treated during the pandemic had higher risk of extra prostatic disease (OR:1.35, $p=0.038$ ) and lymph node invasion (LNI) (OR:1.72, $p=0.048$ ). An average $23 \%$ reduction of the SV with the equivalent timespan of the previous year allowed an illusory reduction of the WL after the peak gained during the first wave of COVID-19. Conclusions Our results showed that the COVID-19 outbreak resulted in a delay in the administration of curative-intent therapies in patients with localized PCa. This, in turn, resulted in a stage migration phenomenon with a potential impact on oncologic control.

Key Words: prostate cancer $\iota$ radical prostatectomy 〈〉COVID-19 〈〉 surgical volume () waiting list «) stage migration 
The COVID-19 outbreak caused a sudden global health emergency. Especially within the first wave, in the attempt to minimize the virus transmission, many outpatient and procedural attendances were postponed or canceled with the aim to safeguard patients and healthcare workers. EAU guidelines faced the new need of allowing the best medical treatment whilst minimizing the risk of transmission within this health crisis [1]. However, prostate cancer $(\mathrm{PCa})$ is characterized by a relatively slow progression and excellent 10-year cancer-specific mortality rates [2] with the majority of the patients presenting with localized disease at diagnosis [3]. Consequently, the adoption of expectant management policies was further encouraged during the first phase of the pandemic period with the goal of postponing active treatment options within 3-6 months, when feasible. In particular, the use of radical prostatectomy (RP) was rationalized using the EAU risk classification tool, age and risk factors for COVID-19 adverse outcomes [4]. In addition, some uncertainties arose regarding the widespread adoption of minimally invasive surgery in the surgical management of $\mathrm{PCa}$ patients, where it has been hypothesized that the pneumoperitoneum typical of laparoscopic or robot-assisted surgery might generate aerosol which could favour the diffusion of the novel coronavirus [5, 6]. Nonetheless, whether all these factors concretely contributed to alter the management of $\mathrm{PCa}$ patients and delay the use of RP still remains undocumented. Of note, the delay in the administration of curative-intent therapies might theoretically result in a stage migration phenomenon with a higher increase in the number of patients with adverse pathologic findings at RP. Hence, we aimed to report the impact of COVID-19 on PCa surgical volumes and waiting list of multiple European Tertiary Referral centers and to investigate whether COVID-19 outbreak increased the risk of aggressive $\mathrm{PCa}$ at final RP histology.

Patients undergoing RP between March $11^{\text {th }} 2020$ (WHO declaration of pandemic) and $31^{\text {st }}$ December 2020 at 8 European urological centers were retrospectively identified from institutional prospective registries. Metastatic and recurrent $\mathrm{PCa}$ cases after non-surgical treatment were excluded. The centers provided both data regarding RPs during pandemic (cases) and records from patients who were treated between March $11^{\text {th }} 2019$ and 31 ${ }^{\text {st }}$ December 2019 which were considered as controls. Data collection included the total numbers of surgeries (i.e., surgical volume [SV]), waiting list (defined as time from biopsy to surgery (WL)), demographic, pre- and postoperative variables.

A total of 927 cases (i.e., patients treated during the COVID19 pandemic period) and 1647 controls (i.e., patients treated the year before) were included. The two groups were similar for most of the demographic and clinical characteristics (Table 1). However, we recorded a lower use of mpMRI, a higher PI-RADS score, cT stage and number of pelvic lymph node dissections during the pandemic phase (all $\mathrm{p}$ values <0.05). At multivariable logistic regression no differences were found for ISUP $4-5$ at pathology, positive surgical margins and use of robotic surgery (Table 2). However, patients being treated during the pandemic had higher risk of extra prostatic disease (OR:1.35, 95\% CI 1.01-1.82, $\mathrm{p}=0.038$ ) and of lymph node invasion (LNI) (OR:1.72, 95\% CI 1.00-2.99, $\mathrm{p}=0.048$ ). Figure 1 depicts the monthly percentage change of SV and WL during pandemic with the equivalent timespan of the previous year. An average of $23 \%$ reduction of the SV was observed. A pro-

Table 1. Patients characteristics

\begin{tabular}{|c|c|c|c|}
\hline & Controls & Cases & $\mathrm{p}$ \\
\hline N. of surgeries & 1647 & 927 & \\
\hline Age, year (median, IQR) & $66.1(61-72)$ & $67(61-72)$ & 0.17 \\
\hline $\mathrm{BMI} \mathrm{kg} / \mathrm{m}^{2}$ (median, IQR) & $26.0(24.1-28.4)$ & $26.3(24.3-28.9)$ & 0.52 \\
\hline $\begin{array}{l}\text { PSA at diagnosis mg/dl } \\
\text { (median, IQR) }\end{array}$ & $7.9(5.5-13.6)$ & $8.4(5.6-13.9)$ & 0.56 \\
\hline Staging with MRI & $741(45.0)$ & $334(36.1)$ & $<0.01$ \\
\hline PI-RADS $\geq 3$ & $543 / 741(78.8)$ & $274 / 334(85.4)$ & 0.01 \\
\hline cT stage $>2$ & 259 (16.9) & $179(21.0)$ & 0.01 \\
\hline $\begin{array}{l}\text { ISUP grade } \\
\text { I } \\
\text { II } \\
\text { III } \\
\text { IV } \\
\text { V }\end{array}$ & $\begin{array}{l}318(19.4) \\
549(33.6) \\
320(19.6) \\
262(16.0) \\
186(11.4)\end{array}$ & $\begin{array}{l}146(16.1) \\
337(37.2) \\
165(18.2) \\
144(15.9) \\
115(12.7)\end{array}$ & 0.13 \\
\hline $\begin{array}{l}\text { Time from biopsy to RP, } \\
\text { months (IQR) }\end{array}$ & $2.6(1.8-4.1)$ & $2.8(1.7-4.3)$ & 0.41 \\
\hline Robotic surgery & 1184 (71.9) & $650(70.2)$ & 0.35 \\
\hline Nerve sparing & $1099(72.8)$ & $648(73.9)$ & 0.55 \\
\hline $\begin{array}{l}\text { ISUP at final pathology } \\
\text { No Tumor } \\
1 \\
2 \\
3 \\
4 \\
5\end{array}$ & $\begin{array}{c}2(0.1) \\
161(9.8) \\
826(50.2) \\
280(17.0) \\
154(9.4) \\
224(13.6)\end{array}$ & $\begin{array}{c}1(0.1) \\
72(8.4) \\
506(54.6) \\
137(14.8) \\
76(8.2) \\
129(13.9)\end{array}$ & 0.29 \\
\hline $\begin{array}{l}\text { pT stage } \\
\text { T0 } \\
\text { T2 } \\
\text { T3 } \\
\text { T4 }\end{array}$ & $\begin{array}{c}2(0.1) \\
902(54.8) \\
736(44.7) \\
7(0.4)\end{array}$ & $\begin{array}{c}1(0.1) \\
499(53.8) \\
421(45.4) \\
6(0.6)\end{array}$ & 0.86 \\
\hline PLND performed & $1063(76.4)$ & $711(80.6)$ & 0.02 \\
\hline Patients with Positive nodes & 113/1063 (10.6) & 79/711 (11.1) & 0.48 \\
\hline
\end{tabular}

All analyses were performed on available data. IQR - interquartile range, BMI - body mass index, ISUP - International Society of Urological Pathology (ISUP) grading of prostate cancer, RP - radical prostatectomy, PLND - pelvic lymph node dissection 
Table 2. Multivariable logistic regression analyses to test the impact of the COVID-19 outbreak on the risk of ISUP 4-5, positive surgical margins, $\geq p T 3 a, p N 1, M+$ and use of robotic surgery, after adjusting for confounders

\begin{tabular}{|c|c|c|c|c|c|c|c|c|c|c|c|c|c|c|c|}
\hline \multirow{2}{*}{ Parameter } & \multicolumn{3}{|c|}{ ISUP 4-5 } & \multicolumn{3}{|c|}{ рТЗа } & \multicolumn{3}{|c|}{ PSM } & \multicolumn{3}{|c|}{$\mathrm{N}+$} & \multicolumn{3}{|c|}{ Robotic surgery } \\
\hline & OR & $95 \% \mathrm{Cl}$ & $\mathrm{p}$ & OR & $95 \% \mathrm{Cl}$ & $p$ & OR & $95 \% \mathrm{Cl}$ & $\mathrm{p}$ & OR & $95 \% \mathrm{Cl}$ & $\mathrm{p}$ & OR & $95 \% \mathrm{Cl}$ & $\mathrm{p}$ \\
\hline $\begin{array}{l}\text { Pandemic } \\
\text { vs no pandemic (ref) }\end{array}$ & 0.9 & $0.69-1.34$ & 0.83 & 1.3 & $1.01-1.82$ & 0.03 & 0.9 & $0.68-1.26$ & 0.63 & 1.7 & $1.00-2.99$ & 0.04 & 0.9 & $0.57-1.66$ & 0.99 \\
\hline Age (cont.) & 1.0 & $1.00-1.05$ & 0.03 & 0.9 & $0.98-1.02$ & 0.9 & 1.0 & $0.98-1.02$ & 0.82 & 0.9 & $0.91-0.99$ & 0.01 & 0.9 & 1.05 & 0.53 \\
\hline PSA (cont.) & 1.0 & 0.99-1.00 & 0.84 & 1.0 & 0.99-1.00 & 0.63 & 1.0 & $0.99-1.00$ & 0.75 & 1.0 & 0.99-1.01 & 0.31 & 1.0 & $0.99-1.01$ & 0.30 \\
\hline $\begin{array}{l}\text { cT stage } \\
(\geq 2 \text { vs }<2)\end{array}$ & 4.6 & $2.24-9.51$ & $<0.01$ & 3.5 & $2.02-6.18$ & $<0.01$ & 1.5 & $0.97-2.56$ & 0.07 & 3.3 & $1.71-6.54$ & $<0.01$ & 0.4 & $0.25-0.94$ & 0.03 \\
\hline $\begin{array}{l}\text { ISUP at biopsy } \\
(>2 \text { vs } \leq 2)\end{array}$ & 3.1 & $2.69-3.77$ & $<0.01$ & 1.5 & $1.35-1.73$ & $<0.01$ & 1.2 & $1.07-1.37$ & $<0.01$ & 2.2 & $1.73-2.85$ & $<0.01$ & 0.6 & $0.55-0.82$ & $<0.01$ \\
\hline PI-RADS (cont) & 1.1 & $0.98-1.44$ & 0.08 & 1.6 & $1.33-1.92$ & $<0.01$ & 1.3 & $1.10-1.62$ & $<0.01$ & 1.7 & $1.08-2.68$ & 0.02 & 0.7 & $0.56-1.09$ & 0.15 \\
\hline
\end{tabular}

ISUP - International Society of Urological Pathology grade; PSA - prostate-specific antigen; PSM - positive surgical margin; $\mathrm{N}+$ - positive lymph nodes

gressive reduction of $\mathrm{SV}$ caused an illusory reduction of the WL after its initial $27 \%$ increase during the earliest COVID-19 wave.

Our findings offer a timely snapshot of patients treated with RP during the COVID-19 pandemic in tertiary referral European Institutions. Alarmingly, as reported by others [7], we document a stage migration phenomenon towards a more advanced stage. A decrease in cancer screening could be a possible explanation for this effect [8]. Delays and/or reduced accuracy in PCa diagnostic pathway, possibly resulting in later presentation and thus in more advanced disease may also have contributed to this shift, and triage of cases with prioritization of surgery in pa- tients with unfavorable characteristics. The lower mpMRI use for staging during pandemic compared to the previous year, well matches with this hypothesis. Interestingly, the use of robotic surgery turned out to be similar before and after the pandemic. Indeed, the earlier discharge favored by minimally invasive surgery and minimizing the within-hospital virus infection/transmission likely overtook possible disadvantages linked to the fear of COVID-19 aerosol generating procedures [9]. At the beginning of the pandemic, PCa surgery room was hampered by hospital reorganization and shortage of healthcare workers and devices. There was an initial increase of the WL which showed subsequent decline

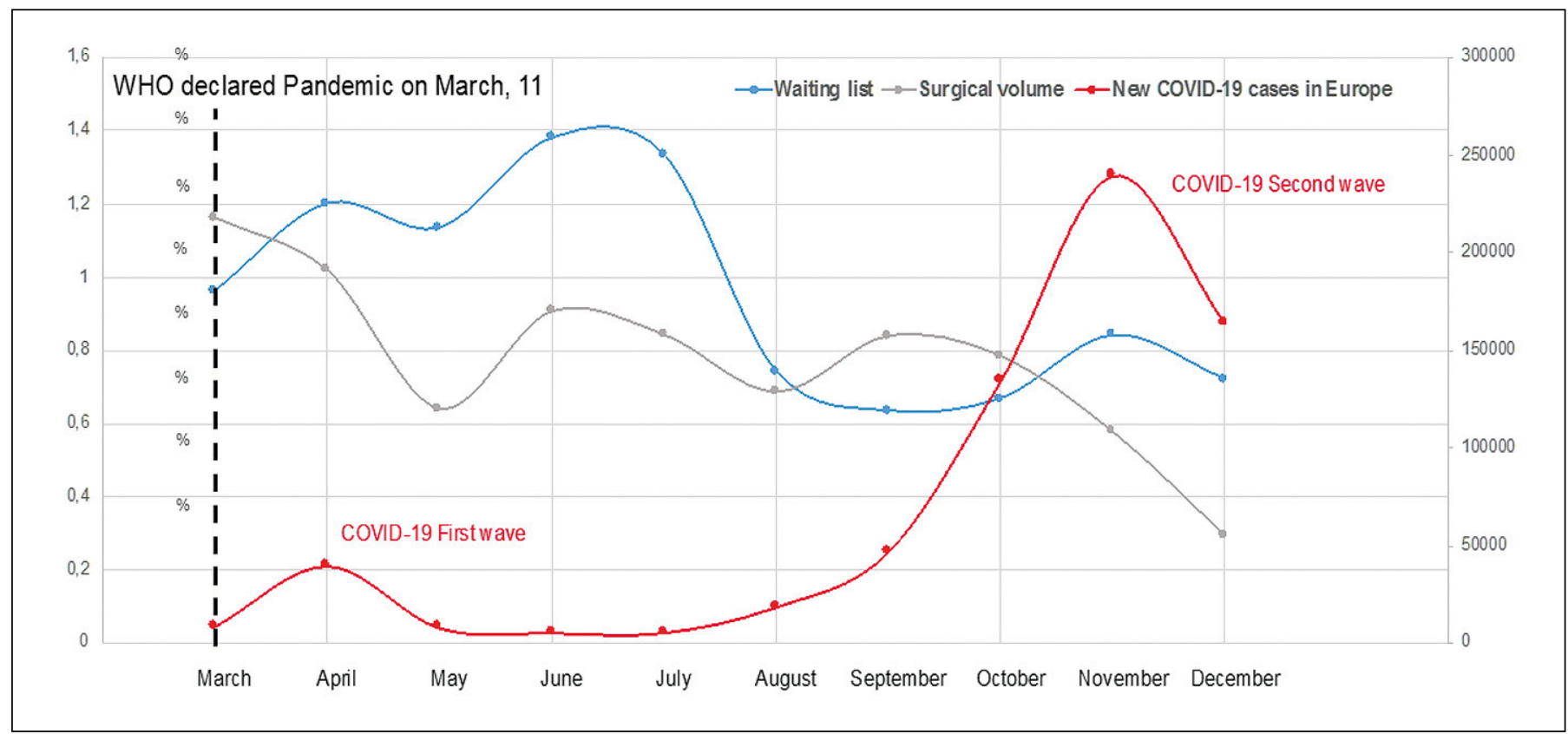

Figure 1. Monthly percentage change of surgical volume and waiting list during the pandemic compared with the equivalent timespan of the previous year. Red line is the superimposed curve of daily increase of new COVID-19 cases in Europe. 
possibly attributable to change in the selection of candidates to RP. Impaired diagnostic paths during the pandemic, may have caused a stage migration towards higher PCa risk categories. Furthermore, it cannot be predicted if an increase of new $\mathrm{PCa}$ diagnosis will be observed when we will revert back from the acute phase of the COVID-19 spread, with more normal levels of urological diagnosis and care. Thus, a new phase of the pandemic may require new strategies to remodulate the SV and WL due to the stage migration and the missing diagnosis. This necessitates a reconfiguration of management pathways and proper selection of candidates for RP. In particular, telemedicine will be able to allow a timely contact with the patients and better counseling; new nomograms might also implement patients selection for the best diagnostic imaging and treatment; hospital reorganization and adapted working schedules are mandatory to guarantee the accessibility to radiological exams and surgical theater; COVID-19-free hospitals dedicated to $\mathrm{PCa}$ might provide the best practice care.

Our findings need to be confirmed by larger cohorts also including other $\mathrm{PCa}$ stages and treatment modalities. The treating physicians could make alternative therapeutic plans to surgery and consider RT or active surveillance as a deliver treatment with minimal to no disruption of the pre COVID treatment timeline. Hence, our results reflect surgical aspects of localized $\mathrm{PCa}$ rather than $\mathrm{PCa}$ features in the pandemic overall.

A false disease state shift cannot be excluded because of decreased surgical volumes and subsequent 'cherry-picking' of higher risk cases; the overall intake disease parameters may in fact be the same but because of lower volumes those patients on the border of needing therapy (eg some intermediate risk patients) may have been delayed in favor of operating on the high risk patient first. Unfortunately, no data can be obtained about those patients still on the waitlist.

Finally, a proper follow up is needed to evaluate if our findings may have implications in the natural history of the disease. There are conflicting data regarding whether postponement/delay of treatment after diagnosis leads to worse outcomes in PCa survivorship [10]. It is likely that the retrospective nature of these studies and heterogeneous patient groups play a large role in the variability of findings. Indeed, the follow up of all cancer patients postponed due to the COVID-19 pandemic will further shed light on impact of treatment delays on PCa survival and disease progression.

\section{CONFLICTS OF INTEREST}

The authors declare no conflicts of interest.

\section{COMPLIANCE WITH ETHICAL STANDARD}

All included patients undergoing radical treatment provided written informed consent for surgery. All procedures performed in studies involving human participants were in accordance with the ethical standards of the institutional and/or national research committee and with the 1964 Helsinki declaration and its later amendments or comparable ethical standards. Institutional review board number was not required due to observational and retrospective nature of the study.

\section{References}

1. Ribal MJ, Cornford P, Briganti A, et al. European Association of Urology Guidelines Office Rapid Reaction Group: An Organisation-wide Collaborative Effort to Adapt the European Association of Urology Guidelines Recommendations to the Coronavirus Disease 2019 Era. Eur Urol. 2020; 78: 21-28.

2. Hamdy FC, Donovan JL, Lane JA, et al. 10-Year Outcomes after Monitoring, Surgery, or Radiotherapy for Localized Prostate Cancer. N Engl J Med. 2016; 375: 1415-1424.

3. Siegel RL, Miller KD, Jemal A. Cancer statistics, 2020. CA Cancer J Clin. 2020; 70: 7-30.

4. Tandogdu Z, Collins J, Shaw G, et al. Management of patients who opt for radical prostatectomy during the COVID-19 pandemic: An International Accelerated Consensus Statement. BJU Int. 2021; 127: 729-741.

5. Pavan N, Crestani A, Abrate A, et al. Risk of Virus Contamination Through Surgical Smoke During Minimally Invasive Surgery: A Systematic Review of the Literature on a Neglected Issue Revived in the COVID-19 Pandemic Era. Eur Urol Focus. 2020; 6: 1058-1069.

6. Mottrie A. ERUS (EAU Robotic Urology Section) guidelines during COVID-19 emergency. 25 March 2020.

7. Roscigno $M$, Naspro R, Piccichè $A$, et al. A Snapshot from the Department of Urology in Bergamo Evaluating the Timeline of the SARS-CoV-2 Outbreak:
Which Patients Are We Missing? Eur Urol Focus. 2020; 6: 1120-1123.

8. Parikh KD, Ramaiya NH, Kikano EG, et al. COVID-19 Pandemic Impact on Decreased Imaging Utilization: A Single Institutional Experience. Acad Radiol. 2020; 27: 1204-1213

9. Motterle G, Dal Moro F, Zanovello N et al. Minimally invasive urologic surgery is safe during COVID-19: experience from two high-volume centers in Italy. J Robot Surg. 2020; 14: 909-911.

10. Cone EB, Marchese M, Paciotti M, et al. Assessment of Time-to-Treatment Initiation and Survival in a Cohort of Patients With Common Cancers. JAMA Netw Open. 2020; 3: e2030072. 